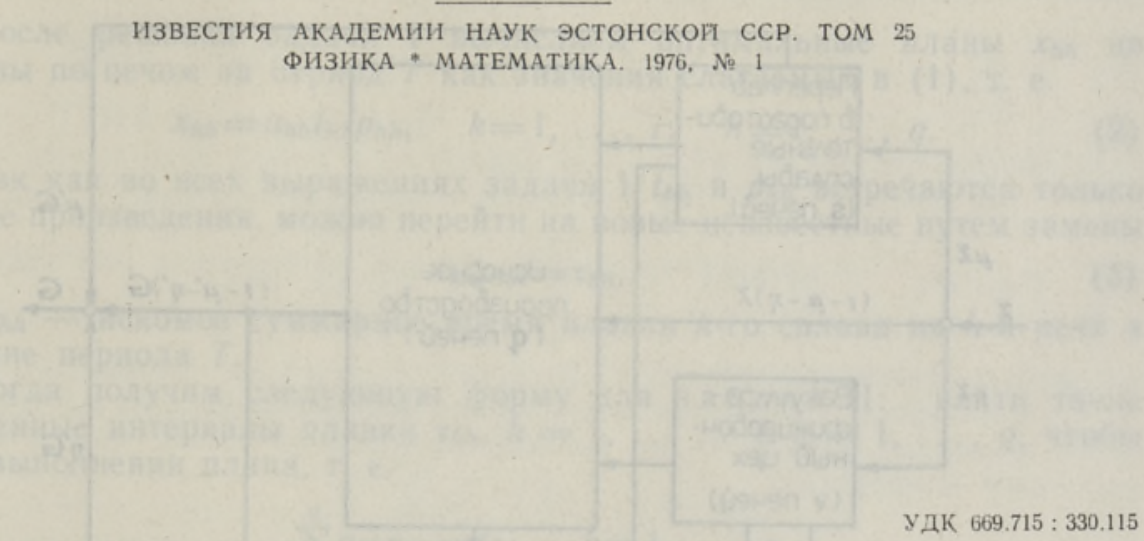

А. ДЬЯЧКО, К. ЖИЛЬЦОВ, Лууле КУУСИК, Надежда САСКОВЕЦ, Марет ТАММ

\title{
ОПТИМАЛЬНОЕ РАСПРЕДЕЛЕНИЕ СЫРЬЯ ПО ПЛАВИЛЬНЫМ АГРЕГАТАМ
}

Специфика производства сплавов из вторичного сырья заключается в тесном взаимодействии шихтового и плавильного переделов. В шихтовых цехах заводов вторичной цветной металлургии сырье подвергается сортировке по видам и разделке для подготовки к плавке и определения хотя бы приблизительного состава металлолома. Поскольку на завод «Вторцветмет» сырье поступает, как правило, смешанное (напр., латунная стружка в смеси с бронзовой или алюминиевой, цинковая стружка в смеси с бесцинковой), определить его химический состав обычно невозможно из-за трудностей отбора достаточно представительной пробы даже при наличии металлургической лаборатории в шихтовом цехе и возможности сплавлять пробы. Поэтому указанное сырье подвергают промежуточному переплаву в шихтовом цехе в т. н. подготовительные сплавы с определенным химическим составом.

Кроме того, сырье, поступающее на заводы вторичной цветной металлургии, содержит большой процент цинка и магния, которые как вредные примеси переходят в готовый продукт. Их концентрации в расплавах можно разбавить лишь большим количеством основы, что экономически нецелесообразно.

В целях рафинирования металла от примесей цинка и магния почти на каждом заводе «Вторцветмет» существует вакуум-рафинировочный цех, оснащенный плавильными агрегатами.

Итак, в плавильное отделение завода поступает сырье после переплава в подготовительные сплавы, сырье после рафинирования, а также сырье со случайным составом, которое можно подвергнуть предварительной сортировке и разделке. Кроме того, промежуточным продуктом является и брак производства.

На рисунке представлена блок-схема производства заводов вторичных цветных металлов с промежуточными продуктами, поступающими из вакуум-рафинировочного цеха и после переплава в подготовительные сплавы.

Перейдем к математической формулировке проблемы оптимального распределения сырья по плавильным агрегатам с учетом только основного производства. Для этого предлагаем следующий подход: разлагая 


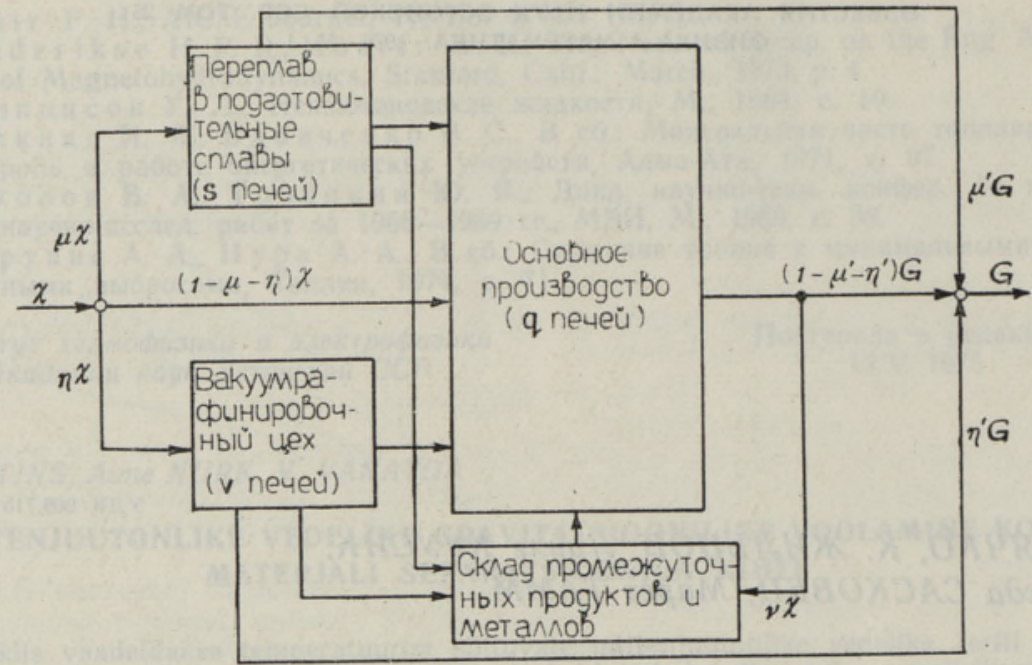

$\chi$ - общее весовое количество вторичного сырья; $\mu$ - часть сырья в процентном отношении, предназначенного для переплава в подготовительный сплав; $\mu^{\prime}$ - часть готовых плановых сплавов, полученных при переплаве в подготовительные сплавы; $\eta$ - часть сырья, прошедшего стадию вакуум-рафинировочного цеха; $\eta^{\prime}$ - часть готовых плановых сплавов, полученных в вакуум-рафинировочном цехе; $v$ доля брака; $1-(\mu+\eta)$ - часть сырья, поступающего непосредственно в основное производство, минуя промежуточные стадии; $G$ общий весовой план по сплавам.

Коэффициенты $\mu, \mu^{\prime}, \eta, \eta^{\prime}$ и $v$ определяются из опытных данных Задачи оптимизации ставятся к части сырья $(1-\mu-\eta) \chi$ и части плана $\left(1-\mu^{\prime}-\eta^{\prime}\right) G$.

проблему на три последовательные задачи линейного программированияя и решая их поочередно, получаем искомый результат.

Во-первых, сформулируем задачу оптимального распределения заданного плана производства сплавов по печам, причем план задается по ассортименту и тоннажу за период $T$ (задача 1$)$.

Введем следующие обозначения:

$G_{k}$ - план на $k$-й вид сплава за период $T, k=1, \ldots, r$;

$a_{k h}$ - производительность $h$-й печи по $k$-му сплаву (весовое количество в единицу времени), $h=1, \ldots, q$;

$t_{k h}$ - период времени, необходимый для производства $k$-го сплава на $h$-й печи (получается из опытных данных);

$p_{k h}$ - искомое число плавок $k$-го сплава на $h$-й печи;

$T_{h}$ - полезное (рабочее) время $h$-й печи в течение периода $T$.

3 адача 1. Найти такое число плавок $p_{k h}, k=1, \ldots, r$ и $h=1, \ldots, q$, чтобы при выполнении номенклатурного плана

$$
\sum_{h=1}^{q} a_{k h} t_{k h} p_{k h}=G_{k}, \quad k=1, \ldots, r,
$$

и ограничении на время

$$
\sum_{h=1}^{r} t_{k h} p_{k h} \leqslant T_{h}, \quad h=1, \ldots, q,
$$

минимизировалось суммарное время плавки

$$
\sum_{k=1}^{r} \sum_{h=1}^{q} t_{k h} p_{k h} \rightarrow \min .
$$


После решения задачи 1 вычисляем оптимальные планы $x_{k h}$ на сплавы по печам за период $T$ как значения слагаемых в (1), т. е.

$$
x_{k h}=a_{k h} t_{k h} p_{k h}, \quad k=1, \ldots, r ; \quad h=1, \ldots, q .
$$

Так как во всех выражениях задачи $1 t_{k h}$ и $p_{k h}$ встречаются только в виде произведения, можно перейти на новые неизвестные путем замены

$$
t_{k h} p_{k h}=\tau_{k h},
$$

где $\tau_{k h}$ - искомое суммарное время плавки $k$-го сплава на $h$-й печи в течение периода $T$.

Тогда получим следующую форму для 3 адачи 1: найти такие временные интервалы плавки $\tau_{k h}, k=1, \ldots, r$ и $h=1, \ldots, q$, чтобы при выполнении плана, т. е.

$$
\sum_{h=1}^{q} a_{k h} \tau_{k h}=G_{k}, \quad k=1, \ldots, r,
$$

и соблюдении временных лимитов, т. е.

$$
\sum_{h=1}^{r} \tau_{k h} \leqslant T_{h}, \quad h=1, \ldots, q,
$$

минимизировалось суммарное время плавки, т. е.

$$
\sum_{k=1}^{r} \sum_{h=1}^{q} \tau_{k h} \rightarrow \min
$$

Задача 1 является типовой задачей линейного программирования, a именно - распределительной задачей.

Оптимальное решение задачи 1 содержит максимально $r+q$ положительных значений $\tau_{k h}, k=1, \ldots, r$ и $h=1, \ldots, q$. Обозначим множество таких пар $(k, h)$, при которых получается. $\tau_{k h}>0$, через $P$, т. е.

$$
P=\left\{(k, h) \mid k \in(1, \ldots, r), h \in(1, \ldots, q), \tau_{k h}>0\right\}
$$

или по (3) и (2)

$$
P=\left\{(k, h) \mid k \in(1, \ldots, r), h \in(1, \ldots, q), x_{k h}>0\right\} .
$$

По формулам (3) и (2) будем иметь из решения задачи 1 не больше чем $r+q$ положительных плановых показателей $x_{k h},(k, h) \in P$.

Во-вторых, сформулируем задачу оптимального распределения вторичного сырья в соответствии с найденными планами (задача 2).

Пусть заданы планы $x_{k h}$ на сплавы по печам и среднестатистический состав вторичного сырья по видам. Требуется определить оптимальные весовые количества шихтовых материалов для каждой печи и каждого сплава при условии выполнения производственного плана по ассортименту и тоннажу.

3 а дач а 2. Найти такое распределение вторичного сырья $x_{k h}^{j}$ по плавильным агрегатам, где $(k, h) \in P, j=1, \ldots, m$, чтобы

$$
Z=\sum_{(k, h) \in P} \sum_{j=1}^{m}\left[c_{k}\left(1-\beta^{j}\right) \gamma_{h}^{j}-c_{h}^{j}\right] x_{k h}^{j} \rightarrow \max ,
$$

где $c_{k}$ - цена единицы $k$-го сплава; $\beta^{j}-$ степень засоренности $j$-го сырья, $\% ; \gamma_{h}^{j}$ - степень извлечения $j$-го сырья из $h$-й печи, $\% ; c_{h}^{j}-$ цена единицы $j$-го сырья вместе с транспортировкой к $h$-й печи, при условиях:

а) брутто-вес $j$-го сырья, загружаемого в $h$-ю печь, должен удовлетворять составу $k$-го сплава по ГОСТу: 


$$
\begin{aligned}
& \sum_{j=1}^{m}\left(\alpha_{i}^{j}-\alpha_{i k}\right)\left(1-\beta^{j}\right) \gamma_{h}^{j} x_{k h}^{j} \geqslant 0, \\
& \sum_{j=1}^{m}\left(\bar{\alpha}_{i k}-\alpha_{i}^{j}\right)\left(1-\beta^{j}\right) \gamma_{h}^{j} x_{k h}^{j} \geqslant 0,
\end{aligned}
$$

где $\alpha_{i k}\left(\overline{\alpha_{i k}}\right)-$ нижний (верхний) предел содержания $i$-го элемента в $k$-ом сплаве (по ГОСТу), $\% ; \boldsymbol{\alpha}_{i}^{j}-$ содержание $i$-го элемента в $j$-ом сырье (по опытным данным), \%;

б) суммарное количество $j$-го сырья, необходимое для выполнения всего плана за период $T$, не должно превышать запаса сырья $B^{j}$ на шихтовой площадке:

$$
\sum_{(k, h) \in P} x_{k h}^{j} \leqslant B^{j}, \quad j=1, \ldots, m
$$

в) суммарный выход каждого сплава из каждой печи должен превышать плановый $x_{k h}$ или быть равным ему:

$$
x_{k h} \leqslant \sum_{j=1}^{m}\left(1-\beta^{j}\right) \gamma_{h}^{j} x_{k h}^{j}, \quad(k, h) \in P ;
$$

г) общее количество сырья, перерабатываемое в $h$-й печи в $k$-й сплав за период $T$, не должно превышать максимальной печной емкости $\bar{D}_{h}$, умноженной на число плавок $p_{k h}$, которое вычисляется по (3):

$$
\sum_{j=1}^{m} x_{k h}^{j} \leqslant p_{k h} \bar{D}_{h}, \quad(k, h) \in P .
$$

Решив задачу 2, получим распределение сырья, имеющегося на складе в начале периода $T$, по печам в соответствии с планом.

В дальнейшем используем запас $j$-го сырья для $h$-й печи, который получается из предыдущего решения:

где

$$
B_{h}^{j}=\sum_{k \in K_{h}} x_{k h}^{j}, \quad j=1, \ldots, m \quad \text { и } h \in H,
$$

$$
\begin{gathered}
K_{h}=\{k \mid k \in(1, \ldots, r),(k, h) \in P\}, \\
H=\{h \mid h \in(1, \ldots, q), \quad(k, h) \in P\} .
\end{gathered}
$$

В-третьих, для каждой фиксированной печи приступим к расчетам шихты по каждой плавке. Сформулируем задачу 3 - задачу оптимального расчета шихты по каждой плавке для фиксированной $h$-й печи.

3 ад ач а 3. Найти такое количество сырья $x_{k h}^{j l}, \quad k \in K_{h}, j \in J_{h}$, где

$$
J_{h}=\left\{j \mid j=1, \ldots, m, B_{h}^{j}>0\right\},
$$

загружаемое в $h$-ю печь для $l$-й плавки, $l=1, \ldots, p_{k h}$, чтобы

$$
Z_{h}=\sum_{j \in J_{h}} \sum_{k \in K_{h}}\left[c_{k}\left(1-\beta^{j}\right) \gamma_{h}^{j}-c_{h}^{j}\right] \sum_{l=1}^{p_{k h}} x_{k h}^{j l} \rightarrow \max
$$

при условиях:

а) брутто-вес сырья по $l$-й плавке, загружаемого в $h$-ю печь, должен удовлетворять составу по ГОСТу:

$$
\sum_{j \in J_{h}}\left(\alpha_{i}^{j}-\underline{\alpha}_{i k}\right)\left(1-\beta^{j}\right) \gamma_{h}^{j} x_{k h}^{j l} \geqslant 0, \quad i=1, \ldots, n, \quad k \in K_{h},
$$




$$
\sum_{j \in J_{h}}\left(\bar{\alpha}_{i k}-\alpha_{i}^{j}\right)\left(1-\beta^{j}\right) \gamma_{h}^{j} x_{k h}^{j l} \geqslant 0, \quad l=1, \ldots, p_{k h} ;
$$

б) суммарный выход $k$-го сплава по ГОСТу на фиксированной печи за период $T$ должен превышать плановый или быть равным ему:

$$
x_{k h} \leqslant \sum_{j \in J_{h}}\left(1-\beta^{j}\right) \gamma_{h}^{j} \sum_{l=1}^{p_{k h}} x_{k h}^{j l}, \quad k \in K_{h} ;
$$

в) суммарное количество каждого расходуемого вида сырья не должно превышать его запаса для данной печи:

$$
\sum_{h \in K_{h}} \sum_{l=1}^{p_{k h}} x_{k h}^{j l} \leqslant B_{h}^{j}, \quad j \in J_{h}
$$

г) общий вес сырья, перерабатываемого в $h$-й печи за $l$-ю плавку, ограничен из-за технологических соображений:

$$
\underline{D}_{h} \leqslant \sum_{j \in J_{h}} x_{k h}^{j l} \leqslant \bar{D}_{h}, \quad k \in K_{h}, \quad l=1, \ldots, p_{k h} .
$$

Если к условиям (5)-(8) добавить требование, что все значения

$$
\xi_{k h}^{j l}=\frac{x_{k h}^{j l}}{g_{h}^{j}}, \quad k \in K_{h}, \quad j \in J_{h}, \quad l=1, \ldots, p_{k h},
$$

есть целые числа, где $g_{h}^{j}$ - объем контейнера (короба) в тоннах $j$-го сырья для $h$-й печи, то путем замены

$$
x_{k h}^{j l}=g_{h}^{j} \xi_{k h}^{j l}, \quad k \in K_{h}, \quad j \in J_{h}, \quad l=1, \ldots, p_{k h},
$$

задача 3 принимает форму задачи линейного целочисленного программирования: найти такие целые числа $\xi_{k h}^{j !}, k \in K_{h}, j \in J_{h}, l=1, \ldots$, $p_{k h}$, чтобы

$$
\zeta_{h}=\sum_{j \in J_{h}} \sum_{k \in K_{h}}\left[c_{k}\left(1-\beta^{j}\right) \gamma_{h}^{j}-c_{h}^{j}\right] g_{h}^{j} \sum_{l=1}^{p_{k h}} \xi_{k h}^{j l} \rightarrow \max
$$

при условиях:

$$
\begin{gathered}
\sum_{j \in J_{h}}\left(\alpha_{i}^{j}-\underline{\alpha}_{i k}\right)\left(1-\beta^{j}\right) \gamma_{h}^{j} g_{h}^{j} \xi_{k h}^{j l} \geqslant 0, \quad i=1, \ldots, n, \quad k \in K_{h}, \\
\sum_{j \in J_{h}}\left(\bar{\alpha}_{i k}-\alpha_{i}^{j}\right)\left(1-\beta^{j}\right) \gamma_{h}^{j} g_{h}^{j} \xi_{k h}^{j l} \geqslant 0, \quad l=1, \ldots, p_{k h}, \\
x_{k h} \leqslant \sum_{j \in J_{h}}\left(1-\beta^{j}\right) \gamma_{h}^{j} g_{h}^{j} \sum_{l=1}^{p_{k h}} \xi_{k h}^{j l}, \quad k \in K_{h}, \\
\sum_{k \in K_{h}} \sum_{l=1}^{p_{k h}} \xi_{k h}^{j l} \leqslant \frac{1}{g_{h}^{j}} B_{h}^{j}, \quad j \in J_{h}, \\
\underline{D}_{h} \leqslant \sum_{j \in J_{h}} g_{h}^{j} \xi_{k h}^{j l} \leqslant \bar{D}_{h}, \quad k \in K_{h}, \quad l=1, \ldots, p_{k h} .
\end{gathered}
$$


Итак, последовательное решение цепочки описанных выше трех задач (вернее, $2+q$ задач, где $q$ - число печей) даст ответ на вопрос об оптимальном распределении сырья по печам и плавкам с учетом плана выпуска сплавов, обеспечивающего экономию дефицитного вторичного сырья и дорогостоящих чистых металлов. Такой способ решения естествен и целесообразен, поскольку он позволяет: 1) значительно сократить размерности решаемых задач линейного программирования, 2) дать компромиссное оптимальное решение проблемы путем применения трех разных целевых функций - минимизации суммарного времени обработки, максимизации валовой продукции в денежном выражении и минимизации затрат на сырье (последние две объединены в максимизацию прибыли).

Описанный комплекс задач является основным звеном в автоматизированной системе управления производством вторичных сплавов цветных металлов. Их применение для составления графиков загрузки печей сырьем предполагается провести с помощью составленных нами программ для ЭВМ «Минск-32» на Мценском заводе «Вторцветмет».

Московский институт стали и сплавов

Государственный научно-исследовательский

Поступила в редакцию институт иветных металлов

Ннститут кибернетики

Академии наук Эстонской ССР

\section{A. DJATSKO, K. ZILTSOV, LuUle KUUSIK,} Nadežda SASKOVETS, Maret TAMM

\section{TOORAINE OPTIMAALNE JAOTAMINE SULATUSAGREGAATIDE VAHEL}

Värvilise metallurgia tehastes kerkib sekundaartoorainest sulamite tootmisel probleem, kuidas toorainet sulatusagregaatide vahel optimaalselt jaotada, et täita või ületada sulamite tootmise plaan võimalikult suure kasumiga, hoides kokku defitsiitseid sekundaartooraineid ja kalleid puhtaid metalle. Probleem tükeldatakse kolmeks järjestikku lahendatavaks, kuid üksteisega seotud lineaarplaneerimisülesandeks (neist esimene on nn. jaotusülesanne ja viimane täisarvuline ülesanne). Koostatud mudelid on värviliste metallide sekundaarsulamite tootmise automatiseeritud juhtimissüsteemi põhilülid.

\section{A. DYACHKO, K. ZHILTSOV, Luule KUUSIK,} Nadezhda SASKOVETS, Maret TAMM

\section{OPTIMAL DISTRIBUTION OF RAW MATERIALS TO SMELTING AGgREGATES}

Optimal distribution problem of secondary raw materials to smelting aggregates for processing nonferrous alloys on remelting plants is considered. The problem is decomposed into three sequential linear programs. The first of these is a distribution problem, the third - an integer one. The objective functions consist of the melt rate, prices of scrap, pure metals, and alloys. The models proposed will be used to manage remelting processes of scrap into nonferrous alloys on remelting plants. 